\title{
A Robust Localization Algorithm for Mobile Sensors Using Belief Functions
}

\author{
Farah Mourad, Hichem Snoussi, Fahed Abdallah, and Cédric Richard, Senior Member, IEEE
}

\begin{abstract}
One of the main objectives of localization algorithms is to compute accurate estimates of sensor positions. This task is usually performed using measurements exchanged with neighboring sensors. However, when erroneous measurements occur, the localization process may yield wrong estimates, which leads to unreliable information for location-based applications. This paper proposes a robust localization technique that works efficiently, even under unreliable measurements assumptions. The proposed method uses belief function theory to estimate sensors locations. Assuming that the reliability of sensors measurements is known, the method combines all the available information to make a final decision about the positions. Each measurement is then used to define a belief function based on the reliability information. Experiments with simulated data demonstrate the effectiveness of this approach compared with state-of-the-art methods using different combination rules.
\end{abstract}

Index Terms-Belief functions, connectivity measurements, distributed estimation, intervals, reliability of sensors.

\section{INTRODUCTION}

$\mathbf{I}$ $\mathrm{N}$ RECENT years, advances in miniaturization, low-power circuit design, and efficient wireless communication have produced a new technological innovation-wireless sensor networks. These networks are composed of a large number of tiny devices that have sensing, computation, and communication capabilities [1]-[3]. The main constraint of such networks is their limited energy reserves, as their power supplies are nonrenewable. The development of sensor networks was first motivated by military applications such as target tracking and battlefield surveillance. Due to many characteristics of sensor networks, they are now used in many civil and industrial applications, such as environment monitoring, vehicle detection, and healthcare applications [4]-[9]. The major advantage of wireless sensor networks remains in their wireless nature since they can be deployed more rapidly and less expensively than wired networks. However, in such networks where no infrastructure exists, sensors are able to move in an uncontrollable manner, and hence, they need to be relocalized. In fact, in most applica-

Manuscript received July 8, 2010; revised December 3, 2010; accepted February 2, 2011. Date of publication February 17, 2011; date of current version May 16, 2011. The review of this paper was coordinated by Prof. A. Jamalipour.

F. Mourad and H. Snoussi are with the Institut Charles Delaunay-LM2S (UMR STMR CNRS 6279), Université de Technologie de Troyes, 10010 Troyes cedex, France (e-mail: farah.mourad@utt.fr; hichem.snoussi@utt.fr).

F. Abdallah is with the Laboratoire HEUDIASYC (UMR CNRS 6599), Université de Technologie de Compiègne, 60205 Compiègne, France (e-mail: fahed.abdallah@hds.utc.fr).

C. Richard is with the Laboratoire FIZEAU (UMR CNRS 6525), Université de Nice Sophia-Antipolis, 06108 Nice cedex 02, France (e-mail: cedric. richard@unice.fr).

Digital Object Identifier 10.1109/TVT.2011.2115265 tions, the measured data are highly related to the geographical location where they have been sensed. For this reason, many researchers in mobile sensors fields have primarily focused on the self-localization of the nodes.

The first solution was to equip all sensors with global positioning systems (GPSs) [10], [11]. Nevertheless, this solution is currently impractical as the GPS receivers are expensive and high energy consuming. For this reason, many alternative algorithms have been proposed to estimate node positions. Most of them consist of equipping a few sensors with GPS receivers. These sensors, called anchors, are aware of their positions. Other sensors, called nonanchor nodes or simply nodes, do not know their positions, and therefore, they exchange information with anchors to be localized. In [12]-[14], anchor-based techniques using the Monte Carlo sequential approach have been proposed [15]. The localization problem is defined using a mobility model as well as the data exchanged between nodes and anchors. The Monte Carlo approach consists of generating a fixed number of positions, called particles, using the localization equations, to cover the solution area. Alternatively, Mourad et al. [16]-[18] performed the localization process using interval analysis [19]-[22]. Based on these techniques, nodes positions are defined as 2-D boxes using intervals.

Almost all previously proposed methods are designed for a trusted environment where no erroneous measurements are considered. However, wireless sensor networks might be deployed in a hostile environment where the strength and the content of exchanged signals can be corrupted. In particular, state-of-the-art techniques use the connectivity measurement approach. It consists of considering an anchor in the vicinity of the considered node if the strength of the signal sent by this anchor and received by the node is higher than a given threshold $\rho_{r}$. A noise or a reflection of the signal could change the corresponding received signal strength indicator (RSSI). Thus, an anchor might be considered within the vicinity of a node while it is not, and vice versa. In these circumstances, the fusion of the observation data could probably lead to an erroneous estimation of nodes positions. In [23], Caron et al. presented a method for data fusion under unreliable sensors assumptions. In this paper, an estimation of the reliability of sensors in addition to a computation of a state vector is performed using particle filters. Different approaches have been proposed for fault diagnosis in the robotic field as well. They are based on very different assumptions, but they are not entirely applicable to the localization problem in mobile sensor networks due to their complexity and their high energy consumption.

The positioning of mobile sensors remains a challenging task within a network where unreliable measurements may 
occur. This paper proposes an original method for position estimation where the confidence in measured data is assumed to be known. The proposed method uses belief theory, also known as the Dempster-Shafer theory, or the evidence theory [24][28]. The information provided by a measure is used to define a belief function. Belief functions are then combined, using belief theory and interval analysis [19], [22] to define a set of regions, each of which having a specific weight. The resulting boxes are then propagated to be used with the future measurements to define the next-step position boxes. The main advantage of the proposed method lies in the consideration of more than one possible solution using the reliability of the measured data. Based on this technique, the proposed method is more robust than the existing methods, particularly in hostile environments.

The rest of this paper is organized as follows. Section II describes the localization problem. The basic tools of the belief theory are then introduced in Section III. In Section IV, the localization algorithm is presented using the belief theory. Simulations are performed in Section V, whereas Section VI concludes the paper.

\section{Problem Statement}

To define the localization problem, two types of sensors are introduced, namely, 1) the anchors and 2) the nonanchor nodes, or simply nodes. Anchors are sensors equipped with a GPS, and thus, they know their positions. Unlike anchors, nodes are unaware of their locations and, hence, exchange information with anchors to localize themselves. Therefore, position estimation is performed using anchors information. Mobile nodes take advantage of their mobility to refine the localization problem, as well. In the following, both the mobility model and the observation equations are introduced.

\section{A. Mobility Model}

The proposed method benefits from the mobility of the nodes to improve the accuracy of the estimation. The mobility equation consists of a prior state-space equation given by

$$
\boldsymbol{x}(t)=\boldsymbol{f}(\boldsymbol{x}(t-1))
$$

where $\boldsymbol{x}(t)$ and $\boldsymbol{x}(t-1)$ are the coordinate vectors of the mobile node at times $t$ and $t-1$, respectively, and $f$ is a function reflecting the prior information about the node movement.

Many mobility models have been proposed in the literature to simulate sensor movements [29], [30]. This paper employs a very general model, inspired by the random walk mobility model [30]. The proposed model assumes that the maximal velocity of the node is known. In other words, no restriction is made on the direction of the motion. The velocity could be random as well but less than its maximal value. Let $v_{\max }$ be the maximal velocity of the tracked node, and let $\Delta t$ be the duration between two following time steps. Then, the distance traveled by the node between time steps $(t-1)$ and $t$ is less than $\Delta t \cdot v_{\max }$. Let $\theta$ and $v$ be the direction and the velocity of the node, respectively, between $(t-1)$ and $t$. Then, the mobility model is given by

$$
\left\{\begin{array}{l}
x_{1}(t)=x_{1}(t-1)+\Delta t \cdot v \cdot \cos (\theta) \\
x_{2}(t)=x_{2}(t-1)+\Delta t \cdot v \cdot \sin (\theta)
\end{array}\right.
$$

where $\theta$ and $v$ are unknown variables included in $[0,2 \pi]$ and $\left[0, v_{\max }\right]$, respectively, and $\boldsymbol{x}(t)=\left(x_{1}(t), x_{2}(t)\right)$ is the coordinate vector of the mobile node at time $t$. Equation (2) could be reformulated as follows:

$$
\left(x_{1}(t)-x_{1}(t-1)\right)^{2}+\left(x_{2}(t)-x_{2}(t-1)\right)^{2}=(\Delta t \cdot v)^{2}
$$

where $v \leq v_{\max }$. If the previous position $\boldsymbol{x}(t-1)$ is punctual, the mobility constraint consists of a disk equation centered on $\boldsymbol{x}(t-1)$ and having $\Delta t \cdot v_{\max }$ as radius. Any prior information about the mobility of the nodes could be used to refine the proposed model.

\section{B. Observation Model}

In addition to its mobility model, a node uses observation information to localize itself. In particular, it collects connectivity information from anchors within its vicinity to estimate its position. These measurements are based on a comparison of the RSSIs. Let $\rho_{i}(t)$ be the RSSI of the signal emitted by the anchor $\boldsymbol{a}_{i}$ and received by the considered node at time $t$. As shown in the Okumura-Hata model [31], [32], which is also called the channel path loss model, $\rho_{i}(t)$ decreases monotonically with the increase of the distance traveled by the signal as follows:

$$
\rho_{i}(t)=\rho_{0}-10 \cdot n_{P} \cdot \log _{10} \frac{d\left(\boldsymbol{a}_{i}(t), \boldsymbol{x}(t)\right)}{d_{0}}+\varepsilon_{i}(t)
$$

where $\boldsymbol{a}_{i}(t)$ is the position of the anchor $\boldsymbol{a}_{i}$ at time $t, \rho_{i}(t)$ is in decibels below $1 \mathrm{~mW}, \rho_{0}$ (in decibels below $1 \mathrm{~mW}$ ) is the strength of the signal measured at a certain distance $d_{0}, n_{P}$ is a path loss parameter, $d\left(\boldsymbol{a}_{i}(t), \boldsymbol{x}(t)\right)$ is the distance between $\boldsymbol{a}_{i}(t)$ and $\boldsymbol{x}_{j}(t)$, and $\varepsilon_{i}(t)$ is the measurement noise. In particular, $\varepsilon_{i}(t)$ is assumed to be a Gaussian noise.

Let $\rho_{r}$ be the strength related to the communication range value $r$. Then, the node compares all the RSSIs of the signals that it receives at time $t$ to $\rho_{r}$. Practically, one is able to fix $\rho_{r}$ to a specific value and then compute $r$ using $\rho_{r}$ and the Okumura-Hata model. Under irregular radio propagation patterns, the maximal value of the communication range could be used. Consider $I^{*}(t) \subseteq\left\{1, \ldots, N_{a}\right\}$ is the set of indices of the anchors emitting the received signals, where $N_{a}$ is the total number of anchors in the network. According to the Okumura-Hata model, for $i \in I^{*}(t)$, if $\rho_{i}(t) \geq \rho_{r}$, then $d\left(\boldsymbol{a}_{i}(t), \boldsymbol{x}(t)\right) \leq r$. In this case, the anchor $\boldsymbol{a}_{i}$ is considered within the vicinity of the node and will be called detected anchor. In the other case, where $\rho_{i}(t)<\rho_{r}, d\left(\boldsymbol{a}_{i}(t), \boldsymbol{x}(t)\right)>r$, and the anchor is not taken into account.

Let $I(t) \subseteq I^{*}(t)$ be the set of indices of all detected anchors. Each anchor listed in $I(t)$ defines a connectivity measurement that could be used in the localization of the considered node. In fact, all detected anchors are located at a distance from the node less than $r$. Then, for each detected anchor $\boldsymbol{a}_{i}, i \in I(t)$, an observation constraint is set as follows:

$$
\left(x_{1}(t)-a_{i, 1}(t)\right)^{2}+\left(x_{2}(t)-a_{i, 2}(t)\right)^{2} \leq r^{2} .
$$

Therefore, the observations are modeled as equations of disks centered on the detected anchors and having $r$ as radii.

In practice, the RSSIs of exchanged signals could be modified due to an imperfect environment or sensor damages. 
This might lead to erroneous measurements, where anchors are assumed to be within the vicinity of the node while they are not, and vice versa. Let $\rho_{i}^{*}(t)$ and $\rho_{i}(t)$ be the theoretical and the measured RSSIs, respectively, related to a distance $d\left(\boldsymbol{a}_{i}(t), \boldsymbol{x}(t)\right)$. The following four cases could be encountered.

Case 1: $\rho_{i}(t) \geq \rho_{r}$ and $\rho_{i}^{*}(t) \geq \rho_{r}$. In this case, the anchor $\boldsymbol{a}_{i}$ is detected, which is true, leading to an accurate measurement.

Case 2: $\rho_{i}(t)<\rho_{r}$ and $\rho_{i}^{*}(t)<\rho_{r}$. In this case, the anchor $\boldsymbol{a}_{i}$ is withdrawn. This is also true, and no measurement is generated.

Case 3: $\rho_{i}(t)<\rho_{r} \leq \rho_{i}^{*}(t)$. In this case, the anchor $\boldsymbol{a}_{i}$ is supposed out of the detection area of the node, which is false. No measurement is generated, which leads to a loss in the estimation accuracy.

Case 4: $\rho_{i}^{*}(t)<\rho_{r} \leq \rho_{i}(t)$. In this case, the anchor $\boldsymbol{a}_{i}$ is supposed within the vicinity of the node while it is not. A false observation constraint is then obtained, leading to inaccurate estimation of nodes positions. In such a case, the resulting measurements are called erroneous measurements.

The proposed method takes the erroneous measurements into account. Therefore, it assumes that the amounts of confidence that could be given to observations are provided. Let $\beta_{i} \in[0,1]$, $i \in I(t)$ be the reliability values given to the measurements. In particular, $\beta_{i}=1$ means that the measurement is totally trustworthy, whereas $\beta_{i}=0$ means that the measurement is absolutely unreliable. Then, if a detected anchor $\boldsymbol{a}_{i}$ is considered with a reliability equal to $\beta_{i}$, the node is located within the disk centered on the anchor and having $r$ as radius with a reliability equal to $\beta_{i}$. Thus, a correct measurement should have high reliability, whereas an erroneous one should have low reliability. The observation model then consists of the observation equation (5) and its corresponding reliability values.

Remark 1: This paper does not develop a method for reliability evaluation. However, it suggests an evaluation technique based on the RSSI values. According to this technique, the measurement is assumed reliable as much as its RSSI is higher than $\rho_{r}$. In fact, when the RSSI $\rho_{i}(t)$ of a signal is much higher than $\rho_{r}$, there is a high probability to have its theoretical value $\rho_{i}^{*}(t)$ higher than $\rho_{r}$. Let $\beta_{r}$ and $\bar{\beta}$ be the smallest and the highest reliability values, respectively, that are able to be assigned to the measurements. These values correspond, respectively, to the threshold $\rho_{r}$ and a maximal strength $\bar{\rho}$. $\beta_{r}$ and $\bar{\beta}$ could be taken equal to 0 and 1 , respectively. The strength $\bar{\rho}$ could be equal to the initial transmission strength or a multiple of $\rho_{r}$. In all cases, $\bar{\rho}$ should be higher than the maximum of the received strengths $\bar{\rho} \geq \max _{i \in I(t)}\left\{\rho_{i}(t)\right\}$. Therefore, according to this technique, the reliability given to an observation $i$ having an RSSI equal to $\rho_{i}(t)$ could be computed as follows:

$$
\beta_{i}=\beta_{r}+\left(\rho_{i}(t)-\rho_{r}\right) \cdot \frac{\bar{\beta}-\beta_{r}}{\bar{\rho}-\rho_{r}} .
$$

Note that the proposed method remains a suggestion. Other techniques, mainly based on channel analysis, could be used for reliability computation. In addition, in the simulation section, an evaluation of the sensitivity of the proposed method to wrong reliability values assigned to measurements is performed.

\section{BELIEF THEORY}

The belief theory is a branch of mathematics that deals with evidence combination for decision making. This theory has been used in many fields such as data fusion and pattern recognition [33], [34]. This paper uses the belief theory for resolving the localization problem. In this section, the basic definitions of the belief theory are first introduced. The main concepts of evidence combination are then defined.

\section{A. Basic Definitions}

The belief theory, which is also called the Dempster-Shafer theory or the evidence theory, is a variant of the probability theory where elements are not single points but rather sets or intervals [24]-[28]. One fundamental function of the Dempster-Shafer theory is the basic belief assignment (BBA) or simply mass function $m$. Let $\Omega=\left\{\omega_{1}, \ldots, \omega_{n}\right\}$ be the domain of a variable $x$ and let $2^{\Omega}$ be the set of all subsets of $\Omega, 2^{\Omega}=\left\{\emptyset,\left\{\omega_{1}\right\}, \ldots, \Omega\right\} . \emptyset$ denotes the impossible event if $\Omega$ covers all possible values of $x$. Otherwise, it includes all the possible values of $x$ not listed in $\Omega$. For instance, let $x$ be a real variable $\Omega \subseteq \mathbb{R}$. If $\Omega=\mathbb{R}$, then $\emptyset$ denotes the impossible event, whereas if $\Omega \subsetneq \mathbb{R}$, then $\emptyset$ refers to $\bar{\Omega}=\mathbb{R} \backslash \Omega$. $\Omega$ is called the frame of discernment. A mass function defined on $\Omega$, which is denoted by $m^{\Omega}$, is a mapping from $2^{\Omega}$ to the interval $[0,1]$ as follows:

$$
m^{\Omega}: A \in 2^{\Omega} \longmapsto m^{\Omega}(A) \in[0,1]
$$

where $\sum_{A \in 2^{\Omega}} m^{\Omega}(A)=1$. The value of the BBA given to $A$ consists of the proportion of evidence saying that the observed variable $x$ belongs to $A$. The value of $m^{\Omega}(A)$ gives no additional information about any subset of $A$. Let $A_{i}, i \in$ $\{1, \ldots, p\}$, be the subsets of $\Omega$ having nonzero mass values. $A_{i}$, $i \in\{1, \ldots, p\}$, represents the focal elements of the proposed BBA. Particular BBAs exist according to the number or the types of their focal elements. For instance, a BBA $m^{\Omega}$ is called categorical if it has only one focal element $(p=1)$. It is called empty if its only focal element is $\Omega$. Moreover, $m^{\Omega}$ is said to be normal if $m^{\Omega}(\emptyset)=0$.

Two other important notions in the evidence theory are the belief and the plausibility functions. These functions, denoted by bel and $p l$, respectively, are derived from the BBA. Given the BBA function $m^{\Omega}$ introduced above, the belief of a set $A$ is the sum of the BBA values of all the proper subsets of $A$ as follows:

$$
b e l^{\Omega}(A)=\sum_{i \mid A_{i} \subseteq A, A_{i} \neq \emptyset} m^{\Omega}\left(A_{i}\right)
$$

whereas the plausibility of $A$ is the sum of the BBA values of all subsets intersecting $A$ as follows:

$$
p l^{\Omega}(A)=\sum_{i \mid A_{i} \cap A \neq \emptyset} m^{\Omega}\left(A_{i}\right) .
$$

The belief is then the proportion of available evidence given to $A$, while the plausibility is a measure of the maximum support of evidence that could be given to $A$. The probability of the 
TABLE I

EXAMPLE OF A BBA WiTH THE CORRESPONDING Belief ANd PlausibliLity Values Assigned To EACH SubSET of $\Omega=\left\{\omega_{1}, \omega_{2}, \omega_{3}\right\}$

\begin{tabular}{|c|c|c|c|}
\hline$A \in 2^{\Omega}$ & $m^{\Omega}$ & bel $^{\Omega}$ & $p l^{\Omega}$ \\
\hline$\emptyset$ & 0 & 0 & 0 \\
\hline$\left\{\omega_{1}\right\}$ & 0.09 & 0.09 & 0.51 \\
\hline$\left\{\omega_{2}\right\}$ & 0.31 & 0.31 & 0.66 \\
\hline$\left\{\omega_{3}\right\}$ & 0.1 & 0.1 & 0.37 \\
\hline$\left\{\omega_{1}, \omega_{2}\right\}$ & 0.23 & 0.63 & 0.9 \\
\hline$\left\{\omega_{1}, \omega_{3}\right\}$ & 0.15 & 0.34 & 0.69 \\
\hline$\left\{\omega_{2}, \omega_{3}\right\}$ & 0.08 & 0.49 & 0.91 \\
\hline$\left\{\omega_{1}, \omega_{2}, \omega_{3}\right\}$ & 0.04 & 1 & 1 \\
\hline
\end{tabular}

TABLE II

EXAMPLE OF A BBA DEFINED ON $\mathbb{R}$

\begin{tabular}{|c|c|c|c|}
\hline$A_{i}$ & $m^{\Omega}\left(A_{i}\right)$ & bel $^{\Omega}\left(A_{i}\right)$ & $\mathrm{pl}^{\Omega}\left(A_{i}\right)$ \\
\hline$[0,1]$ & 0.2 & 0.2 & 0.6 \\
\hline$[-2,-1]$ & 0.15 & 0.15 & 0.5 \\
\hline$[-3,2]$ & 0.1 & 0.45 & 1 \\
\hline$[-4,-1]$ & 0.25 & 0.4 & 0.5 \\
\hline$[0,5]$ & 0.3 & 0.5 & 0.6 \\
\hline
\end{tabular}

event $A$ falls within an interval whose lower and upper bounds are given by the belief and the plausibility values, respectively. Table I shows an example of a normal BBA, defined on $\Omega=$ $\left\{\omega_{1}, \omega_{2}, \omega_{3}\right\}$. It also shows the belief and the plausibility values assigned to each subset of $\Omega$.

In general, the frame of discernment is assumed to be finite. However, the belief theory could be extended to the infinite case [35], [36]. If $\Omega=\mathbb{R}$, focal elements become intervals [19], [22]. In a multidimensional space, where $\Omega=\mathbb{R}^{n}$, focal elements are multidimensional intervals, also called boxes, rather than real intervals. Table II shows an example of a normal BBA, defined on $\Omega=\mathbb{R}$, with the corresponding belief and plausibility values. The first column of the table gives only the focal elements of the BBA.

Note that when the source of information is not completely reliable, one is able to discount the BBA using a discounting coefficient $(1-\alpha) \in[0,1]$. This coefficient consists of the amount of reliability of the source [27]. Using such coefficient allows us to transfer a part of the evidence to the whole frame $\Omega$. A discounted BBA is obtained as follows:

$$
m^{\alpha, \Omega}(A)= \begin{cases}(1-\alpha) \cdot m^{\Omega}(A), & \text { if } A \in \Omega, A \neq \Omega \\ \alpha+(1-\alpha) \cdot m^{\Omega}(A), & \text { if } A=\Omega .\end{cases}
$$

\section{B. Evidence Combination}

The combination of evidence consists of the aggregation of information coming from multiple sources. These sources provide different assessments for the same frame of discernment. Let $S_{1}$ and $S_{2}$ be two independent sources of information about a variable $x$. The knowledge held by these distinct sources could be quantified by two mass functions $m_{1}$ and $m_{2}$. Let $\mathcal{F}_{1}=\left\{A_{1}, \ldots, A_{p}\right\}$ and $\mathcal{F}_{2}=\left\{B_{1}, \ldots, B_{q}\right\}$ be the sets of focal elements of $m_{1}$ and $m_{2}$, respectively. To combine these sources, different combination rules have been proposed, such as the conjunctive and the disjunctive rules [37], [38]. In this paper, the conjunctive rule of combination is used since it leads to a more informative and specialized BBA. The combination of the information provided by both sources using the conjunctive rule yields a mass function $m_{1 @ 2}$ defined as follows:

$$
m_{1 @ 2}(C)=\left(m_{1} @ m_{2}\right)(C)=\sum_{i, j \mid A_{i} \cap B_{j}=C} m_{1}\left(A_{i}\right) m_{2}(B j)
$$

for all subsets $C \subseteq \Omega$. The mass $m_{1 \bigcirc 2}(\emptyset)$ assigned to the empty set measures the conflict between the sources. To obtain a normal BBA, one is able to apply the Dempster rule, which consists of the conjunctive rule followed by a normalization phase given by

$$
m_{1} \bigoplus_{2}(C)= \begin{cases}\frac{m_{1 \Theta_{2}}(C)}{1-m_{1 \bigcirc_{2}(\emptyset)},} & \text { if } C \subseteq \Omega, C \neq \emptyset \\ 0, & \text { if } C=\emptyset .\end{cases}
$$

\section{State Estimation Using the Belief Theory}

The goal of state estimation is to compute an accurate estimate of the state of a system using measured data. In this paper, we propose a bounding method for state estimation using the belief theory. In this approach, one attempts to build sets containing all state vectors consistent with measurements. Consider a dynamic system that has an unknown state. Different information about the state could be available such as measurements or state evolution. Having multiple sources of information, the method consists of representing the information brought by each source by a mass function. Once mass functions are defined, resolving the problem aims at combining all mass functions using the conjunctive rule of combination, which is given in (11). This leads to a BBA that has multiple focal elements, each of which having a specific mass. At each iteration, the focal element having the highest weight is chosen. The selected focal element would correspond to the set including the unknown state.

\section{LocAlization Algorithm}

The localization problem is defined using mobility and observation equations introduced in Section II as follows:

$$
\left\{\begin{array}{l}
\left(x_{1}(t)-x_{1}(t-1)\right)^{2}+\left(x_{2}(t)-x_{2}(t-1)\right)^{2}=(\Delta t \cdot v)^{2} \\
\left(x_{1}(t)-a_{i, 1}(t)\right)^{2}+\left(x_{2}(t)-a_{i, 2}(t)\right)^{2} \leq r^{2}, i \in I(t) \\
v \leq v_{\max } .
\end{array}\right.
$$

Each observation constraint is also assigned a reliability measure denoted by $\beta_{i}, i \in I(t)$.

\section{A. Description of the Method}

In this paper, the localization problem is resolved using both the belief theory and the interval analysis [19], [22]. The key idea of the method consists of handling each observation equation as a source of information. Note that the sources are independent since each sensor is able to move and exchange information with the network independently from the others. Hence, at each time step, observation BBAs are defined using measurement constraints. The mobility model is also used to define an additional BBA called mobility BBA. Thus, the number of all elementary BBAs is equal to $|I(t)|+1$. Resolving the problem consists of combining all elementary BBAs in a final one, having several focal elements. The frame of discernment $\Omega$ 


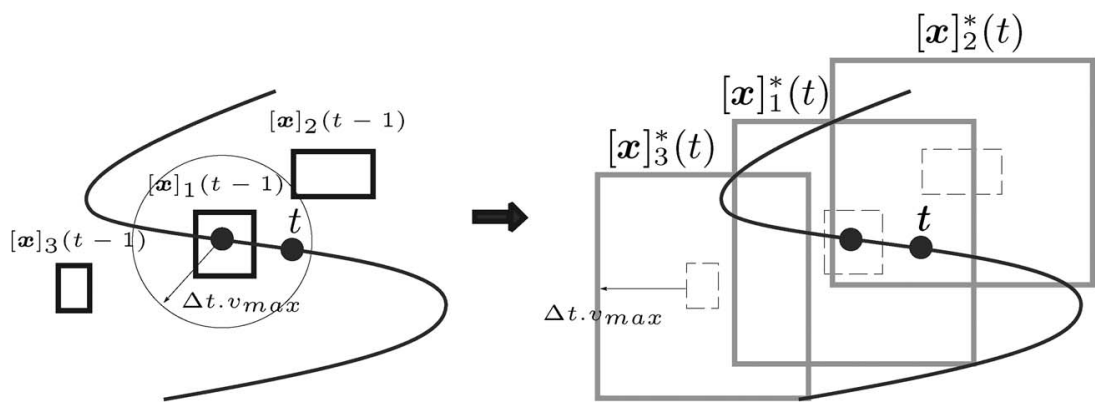

Fig. 1. Generation of the mobility BBA.

is set to the largest area where nodes are able to go. If this area is not bounded, $\Omega$ is set to $\mathbb{R}^{2}$, having all sensors moving in the same plane. Since $\Omega$ is infinite, focal elements of all BBAs will be 2-D intervals [16], [17], [22].

Let $m_{t-1}$ be the final BBA computed at time $t-1$, and let $K(t-1)$ be the number of its focal elements. They are given by $[\boldsymbol{x}]_{k}(t-1)=\left[x_{1}\right]_{k}(t-1) \times\left[x_{2}\right]_{k}(t-1), k \in\{1, \ldots, K(t-$ $1)\}$. The first step of the method consists of propagating these focal elements to the current instant. This step is performed using the mobility model. In the interval framework, all variables are replaced by the intervals at which they belong. In particular, the node coordinate vector $\boldsymbol{x}(t)=\left(x_{1}(t), x_{2}(t)\right)$ is replaced by the box $[\boldsymbol{x}](t)=\left[x_{1}\right](t) \times\left[x_{2}\right](t)[16],[17]$. Then, if (2) is considered, the mobility model is reformulated as follows:

$$
\left\{\begin{array}{l}
{\left[x_{1}\right](t)=\left[x_{1}\right](t-1)+\left[-\Delta t \cdot v_{\max }, \Delta t \cdot v_{\max }\right]} \\
{\left[x_{2}\right](t)=\left[x_{2}\right](t-1)+\left[-\Delta t \cdot v_{\max }, \Delta t \cdot v_{\max }\right] .}
\end{array}\right.
$$

Propagating the previous focal elements using this model leads to the following boxes:

$$
\left\{\begin{array}{l}
{\left[x_{1}\right]_{k}^{*}(t)=\left[x_{1}\right]_{k}(t-1)+\left[-\Delta t \cdot v_{\max }, \Delta t \cdot v_{\max }\right]} \\
{\left[x_{2}\right]_{k}^{*}(t)=\left[x_{2}\right]_{k}(t-1)+\left[-\Delta t \cdot v_{\max }, \Delta t \cdot v_{\max }\right] .}
\end{array}\right.
$$

Since the mobility relation is bijective, masses of the previous focal elements are transmitted to the new boxes. Thus, a mobility BBA $m_{t}^{*}$ could come out of the previous BBA $m_{t-1}$. This BBA has $K(t-1)$ focal elements $[\boldsymbol{x}]_{k}^{*}(t)$, whose masses are given by $m_{t}^{*}\left([\boldsymbol{x}]_{k}^{*}(t)\right)=m_{t-1}\left([\boldsymbol{x}]_{k}(t-1)\right)$. Fig. 1 illustrates the propagation of the BBA $m_{t-1}$ having three focal elements.

In addition to the mobility equation, the observation equations are used to define several mass functions. For each reliable measurement $i, i \in I(t)$, a disk is defined centered on the anchor $\boldsymbol{a}_{i}$ and having the communication range $r$ as radius. This leads to a categorical BBA $m_{i}$ having the disk as a focal element. In the interval framework, each disk $i$ is replaced by the smallest box $A_{i}$ covering it as follows:

$$
\begin{array}{r}
A_{i}=\left[a_{i, 1}(t)-r, a_{i, 1}(t)+r\right] \times\left[a_{i, 2}(t)-r, a_{i, 2}(t)+r\right] \\
i \in I(t) .
\end{array}
$$

Therefore, the BBA corresponding to a reliable measurement $i$ is given by

$$
m_{i}(A)= \begin{cases}1, & \text { if } A=A_{i} \\ 0, & \text { otherwise. }\end{cases}
$$

However, since measurements are not completely reliable, the given BBAs should be discounted according to (10). The discounting coefficients $\alpha_{i}$ are taken equal to $1-\beta_{i}$. The observation BBAs are then given by

$$
m_{i}^{\alpha}(A)= \begin{cases}\beta_{i}, & \text { if } A=A_{i} \\ 1-\beta_{i}, & \text { if } A=\Omega, \\ 0, & \text { otherwise. }\end{cases}
$$

Therefore, a part of the evidence is given to the frame of discernment $\Omega$. In such a case, each observation BBA $m_{i}^{\alpha}$ has two focal elements $A_{i}$ and $\Omega$.

The localization process consists of combining the mobility mass function $m_{t}^{*}$ with all the observation mass functions $m_{i}^{\alpha}$, $i \in I(t)$, according to the conjunctive rule of combination. The final BBA, denoted by $m_{t}$, will be more informative than the elementary ones. To compute $m_{t}$, one should compute its focal elements and then assign them the proper masses. Let $\mathcal{F}^{*}$ and $\mathcal{F}_{i}$ be the sets of focal elements of $m_{t}^{*}$ and $m_{i}^{\alpha}, i \in I(t)$, respectively. Then, $\mathcal{F}^{*}=\left\{[\boldsymbol{x}]_{1}^{*}(t), \ldots,[\boldsymbol{x}]_{K(t-1)}^{*}(t)\right\}$ and $\mathcal{F}_{i}=$ $\left\{A_{i}, \Omega\right\}$. Since $m_{t}$ results from the conjunctive rule of combination, its focal elements are given by the overlapping regions of all possible combinations of the elementary focal elements. Let $\mathcal{C}$ be the combination matrix having $|I(t)|+1$ columns and $K_{\mathcal{C}}$ rows. This special matrix has focal boxes as elements. In other words, the combination number $\ell$, denoted by $\mathcal{C}_{\ell}$ and corresponding to row $\ell$ of $\mathcal{C}$, contains $|I(t)|+1$ elements. In particular, the element number $s$ of $\mathcal{C}_{\ell}$, denoted by $\mathcal{C}_{\ell, s}$, is chosen from the set of focal elements of the sth elementary BBA. Let $I(t)=\left\{i_{1}, \ldots, i_{|I(t)|}\right\}$, then the combination matrix $\mathcal{C}$ is given by the following:

$$
\mathcal{C}=\left(\begin{array}{ccccc}
A_{i_{1}} & A_{i_{2}} & \cdots & A_{i_{|I(t)|}} & {[\boldsymbol{x}]_{1}^{*}(t)} \\
\vdots & \vdots & & \vdots & \vdots \\
A_{i_{1}} & A_{i_{2}} & \cdots & A_{i_{|I(t)|}} & {[\boldsymbol{x}]_{K(t-1)}^{*}(t)} \\
\Omega & A_{i_{2}} & \cdots & A_{i_{|I(t)|}} & {[\boldsymbol{x}]_{1}^{*}(t)} \\
\vdots & \vdots & & \vdots & \vdots \\
\Omega & A_{i_{2}} & \cdots & A_{i_{|I(t)|}} & {[\boldsymbol{x}]_{K(t-1)}^{*}(t)} \\
\vdots & \vdots & & \vdots & \vdots \\
\Omega & \Omega & \cdots & \Omega & {[\boldsymbol{x}]_{1}^{*}(t)} \\
\vdots & \vdots & & \vdots & \vdots \\
\Omega & \Omega & \cdots & \Omega & {[\boldsymbol{x}]_{K(t-1)}^{*}(t)}
\end{array}\right) .
$$

Note that the index $i_{s}, i_{s} \in I(t)$ refers to the anchor number $s$ within the set of the detected anchors over all the anchors in the network. $i_{s}$ is not necessarily equal to $s$. The total number of combinations is given by $K_{\mathcal{C}}=K(t-1) \cdot 2^{|I(t)|}$. Each 


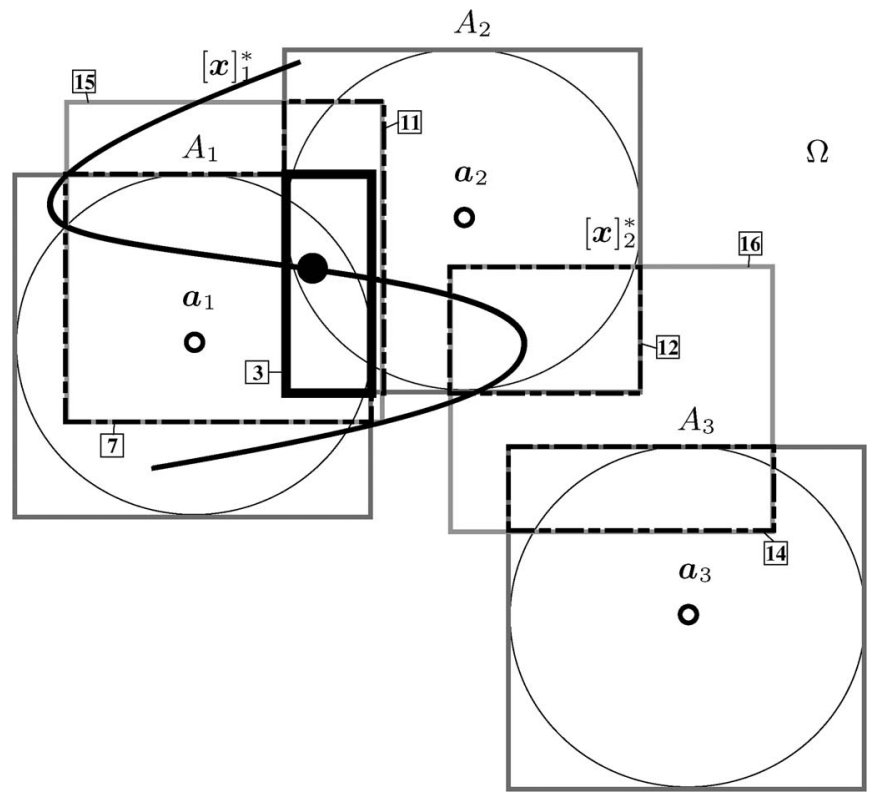

Fig. 2. Example of a localization problem.

combination $\mathcal{C}_{\ell}$ yields a box $B_{\ell}=\bigcap_{s=1}^{|I(t)|+1} \mathcal{C}_{\ell, s}$. According to the combination rule, the mass of a box $B_{\ell}$ is given by

$$
m_{t}\left(B_{\ell}\right)=m_{t}^{*}\left(\mathcal{C}_{\ell,|I(t)|+1}\right) \cdot \prod_{s=1}^{|I(t)|} m_{i_{s}}^{\alpha}\left(\mathcal{C}_{\ell, s}\right) .
$$

Note that in case of conflict, some resulting boxes could be empty. To obtain a normal BBA in such cases, one is able to set to zero the masses of empty boxes and then apply a normalization phase where the sum of all masses is reset to 1.

Each nonempty box $B_{\ell}$ defines a focal element of the normal final BBA. However, since the number of focal elements could be quite large, this method proposes to limit the number of selected focal elements to $K(t)$. This number is either chosen beforehand for all the time steps, or defined instantly by choosing the focal elements having masses higher than a certain bound. The $K(t)$ boxes having the highest masses are then selected to define the focal elements of $m_{t}$, whereas other boxes are withdrawn. A final normalization phase is required in this case. Let $[\boldsymbol{x}]_{k}(t), k \in\{1, \ldots, K(t)\}$ be the final set of focal elements of $m_{t}$. The estimated position at time $t$ is the box $[\boldsymbol{x}]_{k}(t)$ or set of boxes having the highest mass. This box, in addition to the others, will be kept in the memory to be used in the following time step.

Example 1: Consider the localization problem given by three measurements having $\beta_{1}=0.9, \beta_{2}=0.8$, and $\beta_{3}=0.2$. It is also assumed that the previous BBA has two focal elements $[\boldsymbol{x}]_{1}(t-$ 1 ) and $[\boldsymbol{x}]_{2}(t-1)$, whose masses are equal to 0.7 and 0.3 , respectively. Fig. 2 illustrates in gray the focal elements of the elementary BBAs coming out of this problem. The number of all possible combinations is equal to $2 * 2^{3}=16$. Table III shows all possible combinations, the resulting intersection boxes, and their corresponding masses according to the first final BBA $m_{t}^{+}$, where $\emptyset$ has an nonzero mass and the normalized final BBA $m_{t}$. Each nonempty focal element is assigned the corresponding number $\ell$ in Fig. 2. The illustrations show that $m_{t}$ has seven focal elements, which are shown in black, in addition to $[\boldsymbol{x}]_{1}^{*}$ and $[\boldsymbol{x}]_{2}^{*}$, in the plot. The one
TABLE III

Combinations, Focal Elements, And MASSES CorRESPONDING TO THE PROBLEM GIVEN IN Fig. 2

\begin{tabular}{|c|c|c|c|c|}
\hline$\ell$ & $\mathcal{C}_{\ell}$ & $B_{\ell}$ & $m_{t}^{+}\left(B_{\ell}\right)$ & $m_{t}\left(B_{\ell}\right)$ \\
\hline 1 & $A_{1}, A_{2}, A_{3},[\boldsymbol{x}]_{1}^{*}$ & $\emptyset$ & 0.1008 & 0 \\
\hline 2 & $A_{1}, A_{2}, A_{3},[\boldsymbol{x}]_{2}^{*}$ & $\emptyset$ & 0.0432 & 0 \\
\hline 3 & $A_{1}, A_{2}, \Omega,[\boldsymbol{x}]_{1}^{*}$ & $A_{1} \cap A_{2} \cap[\boldsymbol{x}]_{1}^{*}$ & 0.4032 & 0.689 \\
\hline 4 & $A_{1}, A_{2}, \Omega,[\boldsymbol{x}]_{2}^{*}$ & $\emptyset$ & 0.1728 & 0 \\
\hline 5 & $A_{1}, \Omega, A_{3},[\boldsymbol{x}]_{1}^{*}$ & $\emptyset$ & 0.0252 & 0 \\
\hline 6 & $A_{1}, \Omega, A_{3},[\boldsymbol{x}]_{2}^{*}$ & $\emptyset$ & 0.0108 & 0 \\
\hline 7 & $A_{1}, \Omega, \Omega,[\boldsymbol{x}]_{1}^{*}$ & $A_{1} \cap[\boldsymbol{x}]_{1}^{*}$ & 0.1008 & 0.172 \\
\hline 8 & $A_{1}, \Omega, \Omega,[\boldsymbol{x}]_{2}^{*}$ & $\emptyset$ & 0.0432 & 0 \\
\hline 9 & $\Omega, A_{2}, A_{3},[\boldsymbol{x}]_{1}^{*}$ & $\emptyset$ & 0.0112 & 0 \\
\hline 10 & $\Omega, A_{2}, A_{3},[\boldsymbol{x}]_{2}^{*}$ & $\emptyset$ & 0.0048 & 0 \\
\hline 11 & $\Omega, A_{2}, \Omega,[\boldsymbol{x}]_{1}^{*}$ & $A_{2} \cap[\boldsymbol{x}]_{1}^{*}$ & 0.0448 & 0.077 \\
\hline 12 & $\Omega, A_{2}, \Omega,[\boldsymbol{x}]_{2}^{*}$ & $A_{2} \cap[\boldsymbol{x}]_{2}^{*}$ & 0.0192 & 0.033 \\
\hline 13 & $\Omega, \Omega, A_{3},[\boldsymbol{x}]_{1}^{*}$ & $\emptyset$ & 0.0028 & 0 \\
\hline 14 & $\Omega, \Omega, A_{3},[\boldsymbol{x}]_{2}^{*}$ & $A_{3} \cap[\boldsymbol{x}]_{2}^{*}$ & 0.0012 & 0.002 \\
\hline 15 & $\Omega, \Omega, \Omega,[\boldsymbol{x}]_{1}^{*}$ & {$[\boldsymbol{x}]_{1}^{*}$} & 0.0112 & 0.019 \\
\hline 16 & $\Omega, \Omega, \Omega,[\boldsymbol{x}]_{2}^{*}$ & {$[\boldsymbol{x}]_{2}^{*}$} & 0.0048 & 0.008 \\
\hline
\end{tabular}

having the highest weight is $B_{3}$, corresponding to the position estimate. If only focal elements having a mass higher than 0.1 are selected, only two focal elements remain in the list, namely, 1) $B_{3}$ and 2) $B_{7}$. Their final masses after the normalization would be equal to 0.8 and 0.2 , respectively.

\section{B. Alternative Algorithm}

The previous section proposes a method using evidence combination for sensor localization. The presented method relaxes the observation disk equations to define the focal elements of the observation BBAs. Indeed, disks are replaced by the boxes covering them. In this section, an alternative algorithm, allowing us to take disk equations into account, is proposed. This algorithm consists of three steps. Similar to the previous method, the first step corresponds to the propagation phase where the mobility BBA $m_{t}^{*}$ is defined. The second step consists of the combination of the observation measurements only, yielding an observation BBA $m_{o}$. This BBA will be combined to the mobility one afterward in the third step.

Using the belief theory, each measurement having a reliability equal to $\beta$ yields either a disk constraint with a mass equal to $\beta$, or the frame of discernment where the constraint is omitted. Instead of relaxing the disk constraints to define focal elements, the exact equations are employed. For this reason, a binary matrix of combinations is defined where 1 or 0 in a column $s, s \in\{1, \ldots,|I(t)|\}$, means that the constraint $i_{s}$ is selected or not, respectively. $i_{s}$ denotes the $s$ th index in the indices set $I(t)$. Let $\mathcal{C}_{O}$ be this binary matrix; then

$$
\mathcal{C}_{o}=\left(\begin{array}{c}
\mathcal{C}_{o, 1} \\
\mathcal{C}_{o, 2} \\
\vdots \\
\mathcal{C}_{o, K_{o}}
\end{array}\right)=\left(\begin{array}{cccc}
1 & 1 & \cdots & 1 \\
1 & 1 & \cdots & 0 \\
\vdots & \vdots & & \vdots \\
0 & 0 & \cdots & 0
\end{array}\right)
$$

The total number of observation combinations is equal to $K_{o}=$ $2^{|I(t)|}$. A combination $\mathcal{C}_{o, \ell}$ leads to a box $B_{o, \ell}$, resulting of the intersection of all constraints denoted by 1 in $\mathcal{C}_{o, \ell}$. Thus, the mass of a box $B_{o, \ell}$ is given by

$$
m_{o}\left(B_{o, \ell}\right)=\prod_{s=1}^{|I(t)|} q_{\ell, s}
$$


where

$$
q_{\ell, s}= \begin{cases}\beta_{i_{s}}, & \text { if } \mathcal{C}_{o, \ell, s}=1 \\ 1-\beta_{i_{s}}, & \text { otherwise }\end{cases}
$$

In other words, $m_{o}\left(B_{o, \ell}\right)=\prod_{s=1}^{|I(t)|}\left(\beta_{i_{s}} \cdot \mathcal{C}_{o, \ell, s}+\left(1-\beta_{i_{s}}\right)\right.$. $\left.\left(1-\mathcal{C}_{o, \ell, s}\right)\right)$.

For each combination $\ell \leq K_{o}$, the box $B_{o, \ell}$ is obtained by overlapping the disks of the observation constraints assigned a 1 in $\mathcal{C}_{o, \ell}$. Let $J_{\ell}$ be the set of indices of these constraints. To compute the intersection region, each observation equation $i$ is written in terms of intervals as follows:

$$
\left(\left[x_{1}\right](t)-a_{i, 1}(t)\right)^{2}+\left(\left[x_{2}\right](t)-a_{i, 2}(t)\right)^{2}=\left[0, r^{2}\right] .
$$

This constraint could be reformulated as follows [17]:

$$
\left\{\begin{array}{l}
{\left[x_{1}\right](t)=a_{i, 1}(t)+\left[-\bar{b}_{i, 1}(t), \bar{b}_{i, 1}(t)\right]} \\
{\left[x_{2}\right](t)=a_{i, 2}(t)+\left[-\bar{b}_{i, 2}(t), \bar{b}_{i, 2}(t)\right]}
\end{array}\right.
$$

where

$$
\left\{\begin{array}{l}
\bar{b}_{i, 1}(t)=\sqrt{r^{2}-\inf \left(\left(\left[x_{2}\right](t)-a_{i, 2}(t)\right)^{2}\right)} \\
\bar{b}_{i, 2}(t)=\sqrt{r^{2}-\inf \left(\left(\left[x_{1}\right](t)-a_{i, 1}(t)\right)^{2}\right)}
\end{array}\right.
$$

with $\inf ()$ being an operator yielding the inferior bound of the considered interval. The overlapping region is obtained by contracting the whole frame $\Omega$ in the Waltz algorithm using (25). The Waltz contractor [22] is a simple algorithm that iterates all constraints, without any prior order, until no contraction is possible. For each combination $\ell, \Omega$ is reduced to a smaller box covering the intersection region of all disks found in $J_{\ell}$. Using square equations instead of disk equations in this step leads to the same result as with the previous method.

All nonempty observation boxes will be combined afterward with the mobility boxes. The number of all possible combinations is equal to $K(t-1) \cdot K_{o}^{*}$, where $K_{o}^{*} \leq K_{o}$ is the number of nonempty observation boxes. The mass of each resulting box is equal to the product of the observation and the mobility masses of the considered boxes in the combination. Similarly to the previous method, the number of the focal elements of the final BBA $K(t)$ could be limited. Using disk equations, this method leads to smaller focal elements, which increase the estimation accuracy. However, iterating the constraints in the Waltz contractor may be more consuming in terms of computation time. The pseudocode of this method is given in Algorithm 1, where $w()$ yields the width of the considered interval, and $\max _{[K(t)]}\{\}$ gives the $K(t)$ maximal values. In the simulation section, this version of the method will be used.

Note that this alternative method could be easily applied with many different observation models. Indeed, having a whatsoever observation equation, for each combination $\mathcal{C}_{o, \ell}$, all observation constraints assigned a 1 in $\mathcal{C}_{o, \ell}$ are overlapped, leading to an observation box. For instance, one is able to use the RSSI-based model where each measured RSSI is used with the Okumura-Hata model to compute the distance between the anchor sending the signal and the node receiving it. Assume that $d_{i}$ is the computed distance between the anchor $\boldsymbol{a}_{i}$ and the node, and let $\left[d_{i}\right]=\left[d_{i}, \overline{d_{i}}\right]$ be the interval including the incertitude over $d_{i}$. In this case, the observation model is given as follows:

$$
\begin{array}{r}
\left(\left[x_{1}\right](t)-a_{i, 1}(t)\right)^{2}+\left(\left[x_{2}\right](t)-a_{i, 2}(t)\right)^{2}=\left[\frac{d_{i}{ }^{2}}{},{\overline{d_{i}}}^{2}\right] \\
i \in I(t)
\end{array}
$$

where $I(t)$ is the set of indices of all detected anchors. One is able to iterate these constraints in the Waltz algorithm instead of those of disks to compute the observation boxes.

Algorithm 1: Pseudocode of the method.

Input: $K(t),[\boldsymbol{x}]_{k}(t-1), 1 \leq k \leq K(t-1), m_{t-1}, I(t)$, $\boldsymbol{a}_{i}(t), \beta_{i}, i \in I(t), v_{\max }, \Delta t, r$;

Output: $[\boldsymbol{x}]_{k}(t), k \in\{1, \ldots, K(t)\}$;

for $k \in\{1, \ldots, K(t-1)\}$ do

$$
\begin{aligned}
& {\left[x_{1}\right]_{k}^{*}(t)=\left[x_{1}\right]_{k}(t-1)+\left[-\Delta t \cdot v_{\max }, \Delta t \cdot v_{\max }\right] ;} \\
& {\left[x_{2}\right]_{k}^{*}(t)=\left[x_{2}\right]_{k}(t-1)+\left[-\Delta t \cdot v_{\max }, \Delta t \cdot v_{\max }\right] ;} \\
& m_{t}^{*}\left([\boldsymbol{x}]_{k}^{*}(t)\right)=m_{t}\left([\boldsymbol{x}]_{k}(t-1)\right) ;
\end{aligned}
$$

end

$$
K_{o}=2^{|I(t)|}, \quad \mathcal{C}_{o}=\left(\begin{array}{ccc}
1 & \cdots & 1 \\
\vdots & & \vdots \\
0 & \cdots & 0
\end{array}\right) ;
$$

$$
\begin{aligned}
& \text { for } \ell \in\left\{1, \ldots, K_{o}\right\} \text { do } \\
& J_{\ell}=\left\{i_{s} \mid \mathcal{C}_{o, \ell, s}=1, s \in\{1, \ldots,|I(t)|\}\right\} ; \\
& B_{o, \ell}=\Omega \text {; } \\
& A r_{\ell}=w\left(B_{o, \ell, 1}\right) * w\left(B_{o, \ell, 2}\right) A r_{\ell}^{o l d}=A r_{\ell}+1 ; \\
& \text { while } A r_{\ell}<A r_{\ell}^{o l d} \text { do } \\
& A r_{\ell}^{\text {old }}=A r_{\ell} \\
& \text { for } s \in\left\{1, \ldots,\left|J_{\ell}\right|\right\} \text { do } \\
& B_{o, \ell, 2}=B_{o, \ell, 2} \cap\left[a_{i_{s}, 2}(t)-r, a_{i_{s}, 2}(t)+r\right] ; \\
& \bar{b}_{i_{s}, 1}(t)=\sqrt{r^{2}-\inf \left(\left(B_{o, \ell, 2}-a_{i_{s}, 2}(t)\right)^{2}\right)} ; \\
& {\left[\phi_{i_{s}, 1}\right]=a_{i_{s}, 1}(t)+\left[-\bar{b}_{i_{s}, 1}(t), \bar{b}_{i_{s}, 1}(t)\right] ;} \\
& B_{o, \ell, 1}=B_{o, \ell, 1} \cap\left[\phi_{i_{s}, 1}\right] \text {; } \\
& B_{o, \ell, 1}=B_{o, \ell, 1} \cap\left[a_{i_{s}, 1}(t)-r, a_{i_{s}, 1}(t)+r\right] \text {; } \\
& \bar{b}_{i_{s}, 2}(t)=\sqrt{r^{2}-\inf \left(\left(B_{o, \ell, 1}-a_{i_{s}, 1}(t)\right)^{2}\right)} ; \\
& {\left[\phi_{i_{s}, 2}\right]=a_{i_{s}, 2}(t)+\left[-\bar{b}_{i_{s}, 2}(t), \bar{b}_{i_{s}, 2}(t)\right] ;} \\
& B_{o, \ell, 2}=B_{o, \ell, 2} \cap\left[\phi_{i_{s}, 2}\right] \text {; }
\end{aligned}
$$

\section{end}

end

$$
A r_{\ell}=w\left(B_{o, \ell, 1}\right) * w\left(B_{o, \ell, 2}\right) ;
$$

end

$m_{o}\left(B_{o, \ell}\right)=\prod_{s=1}^{|I(t)|}\left(\beta_{i_{s}} \cdot \mathcal{C}_{o, \ell, s}+\left(1-\beta_{i_{s}}\right) \cdot\left(1-\mathcal{C}_{o, \ell, s}\right)\right)$;

$s=0$;

$$
\begin{aligned}
& \text { for } \ell \in\left\{1, \ldots, K_{o}\right\} \text { do } \\
& \text { if } B_{o, \ell} \neq \emptyset \text { then } \\
& \text { for } k \in\{1, \ldots, K(t-1)\} \text { do } \\
& s=s+1,[\boldsymbol{x}]_{s}^{+}(t)=B_{o, \ell} \cap[\boldsymbol{x}]_{k}^{*}(t) ; \\
& \text { end } \\
& m_{t}^{+}\left([\boldsymbol{x}]_{s}^{+}(t)\right)=m_{o}\left(B_{o, \ell}\right) * m_{t}^{*}\left([\boldsymbol{x}]_{k}^{*}(t)\right) \text {; } \\
& \text { end } \\
& \text { end } \\
& S=\left\{s \mid m_{t}^{+}\left([\boldsymbol{x}]_{s}^{+}(t)\right) \in \max _{[K(t)]}\left\{m_{t}^{+}\right\}\right\} ; \\
& \text {for } k \in\{1, \ldots, K(t)\} \text { do } \\
& {[\boldsymbol{x}]_{k}(t)=[\boldsymbol{x}]_{S(k)}^{+}(t), m_{t}\left([\boldsymbol{x}]_{k}(t)\right)=m_{t}^{+}\left([\boldsymbol{x}]_{S(k)}^{+}(t)\right) ;}
\end{aligned}
$$




\section{Simulations}

This section illustrates the performances of the proposed method. All sensors are spread in a $100 \mathrm{~m} \times 100 \mathrm{~m}$ area, which is known as the deployment area. In all simulations, the sensors are assumed to have a group mobility where their movements are dependent on each others [29], [30]. In particular, a group mobility model is generated where sensors are moving in the northeast direction in the first $60 \mathrm{~s}$ and then in the northwest direction until $100 \mathrm{~s}$. An example of the paths of 15 anchors and 30 nodes according to this model is illustrated in Fig. 3. Thus, nodes and anchors are moving for $100 \mathrm{~s}$, the localization period $\Delta t$ being equal to $1 \mathrm{~s}$. Their maximal velocity is equal to $2.2719 \mathrm{~m} \cdot \mathrm{s}^{-1}$, whereas the communication range $r$ is set to $10 \mathrm{~m}$. In the following, an evaluation of the proposed method is first shown under imperfect circumstances. An illustration of the sensitivity of the effectiveness of the method to the reliability values of erroneous measurements is then given. The proposed approach is compared afterward with two different methods based on the Monte Carlo approach and the interval theory, both of them having been developed for a perfect environment. In all simulations, without loss of generality, a single mobile node is considered since it localization problem does not depend on other nodes. Note that the simulations are performed on an Intel(R) Core(TM)2 central processing unit $(2.40 \mathrm{GHz}, 1.00 \mathrm{~GB}$ random access memory) using MATLAB 6.1.

\section{A. Evaluation of the Proposed Method}

To illustrate the effectiveness of the proposed method, ten anchors are randomly deployed, four of them being initially chosen within the vicinity of the node. The others are taken within the ring centered on the node and having $r$ and $2 r$ as radii, where the communication range $r$ is equal to $10 \mathrm{~m}$. In such a case, a high number of erroneous measurements could be obtained, since anchors that are out of the vicinity of the node remain so close to it. Indeed, their corresponding RSSIs, being less than but close to the threshold $\rho_{r}$, are able to overpass it due to a small additive noise. All sensors are supposed to have a group mobility according to the trajectories illustrated in Fig. 3. To generate measurements, the proposed technique proceeds as follows. RSSI values are first generated using the distances between the anchors and the node. This step is performed using a noiseless Okumura-Hata model with $\rho_{o}=100 \mathrm{dBm}$, $d_{0}=0.1 \mathrm{~m}$, and $n_{P}=3$. These parameters yield a threshold $\rho_{r}=40 \mathrm{dBm}$. A Gaussian noise with mean $\mu=0 \mathrm{dBm}$ and variance $\sigma^{2}=4 \mathrm{dBm}^{2}$ is then added to the computed RSSIs. To define the connectivity measurements, the resulting values are compared with $\rho_{r}$. Fig. 4 illustrates the number of detected anchors using the noisy RSSIs. In perfect environments, four anchors are in the vicinity of the node at each time step. In this section, correct measurements are assigned random reliability values taken within the interval $[0.5,1]$, whereas erroneous ones are assigned values within the interval $[0,0.5]$. The reliability values assigned to correct and erroneous measurements are illustrated in Fig. 5. The number of focal elements chosen at each time step is also limited by only keeping those whose

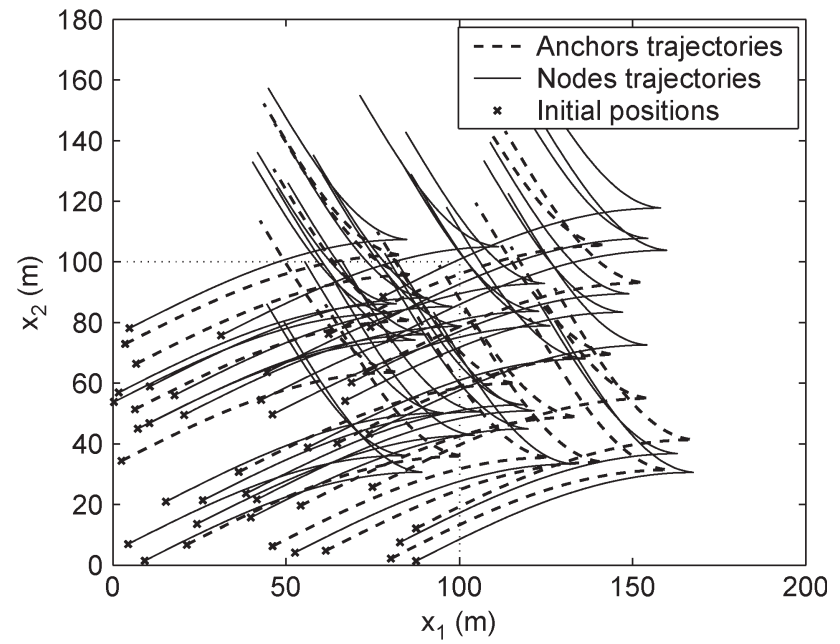

Fig. 3. Paths of 15 anchors and 30 nodes moving together.

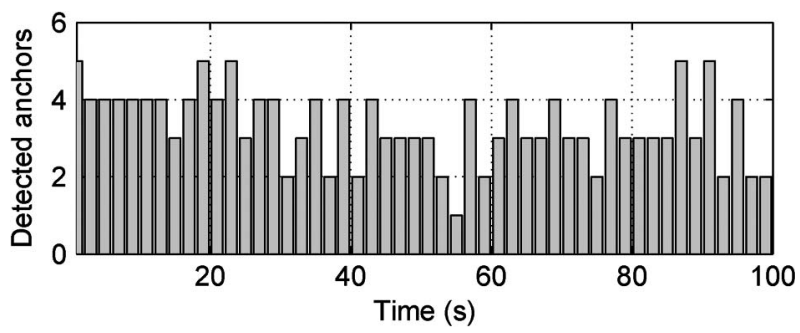

Fig. 4. Number of detected anchors using noisy measurements, shown at odd time steps.

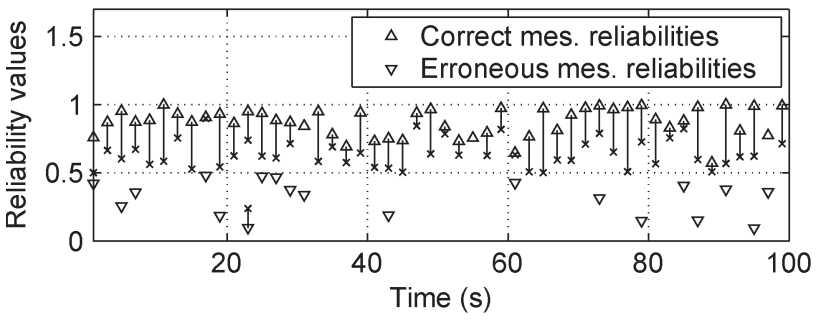

Fig. 5. Reliability values assigned to correct and erroneous measurements, shown at odd time steps.

masses are higher than 0.1. Fig. 6 shows the boxes obtained using our method. The plot shows in straight black lines the boxes with the highest masses, whereas other focal elements are shown in dashed gray lines. At time $t=7 \mathrm{~s}$ for instance, three focal elements are obtained having masses equal to 0.5700 , 0.2627 , and 0.1673 . The estimated position at this time step is the best focal element having the highest mass equal to 0.5700 . The average computation time needed in our method per time step is equal to $0.02433 \mathrm{~s}$. Let the estimation error be the distance between the real position and the center of the box having the highest mass. Then, the average estimation error per time step in this example is equal to $0.4973 \mathrm{~m}$. It is obvious that the estimated boxes include the real positions at all the time steps, which corroborates the effectiveness of the method, even under imperfect circumstances. 


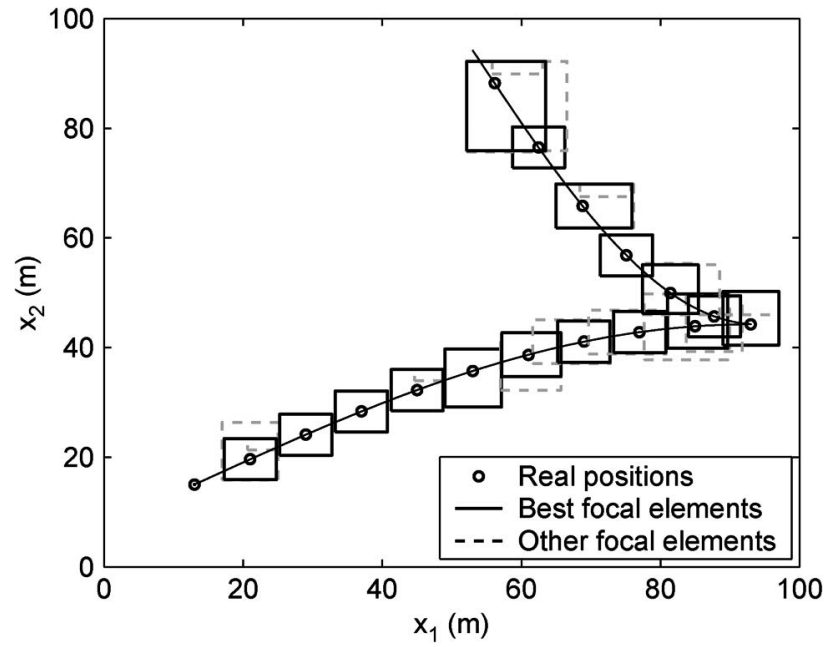

Fig. 6. Boxes obtained using our method.
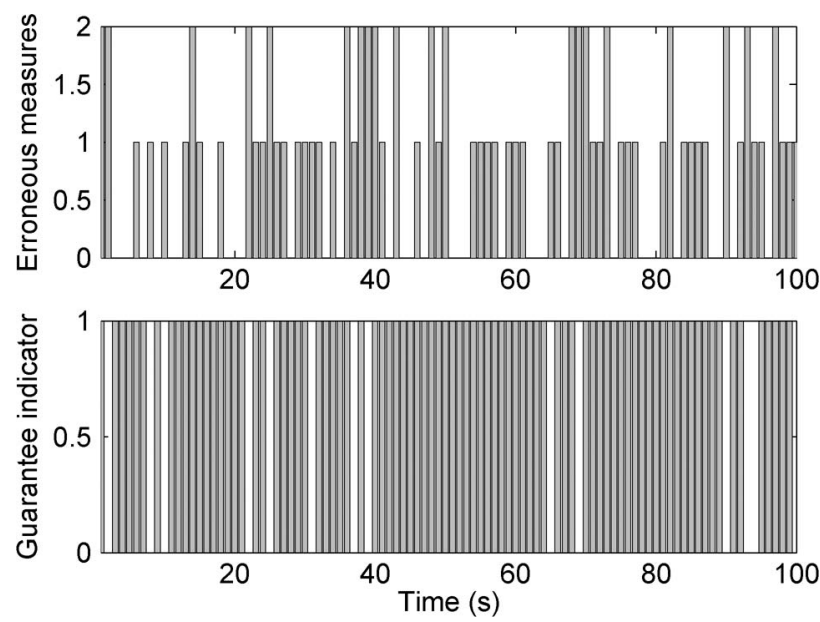

Fig. 7. Number of erroneous measurements on the top plot and the guarantee indicator on the bottom plot.

\section{B. Sensitivity to the Reliability of Measurements}

In the previous section, erroneous measurements are assigned low reliability values (less than 0.5 ), whereas correct ones are given high reliability values (higher than 0.5 ). Under these circumstances, the guaranteed aspect of the method is maintained. In other words, the real position is included in the estimated box at each time step. In this section, it is assumed that the model used to assign the reliability values to measurements is irrelevant. Thus, erroneous measurements could have high reliability values and maybe higher than those of good measurements. First, all erroneous measurements are assumed to have high reliability values taken within [0.5, 0.75]. In this example, the correct measurements have always higher reliability values than the erroneous ones. Their values are chosen within $[0.75,1]$. The top plot of Fig. 7 shows the number of erroneous measurements created at each time step. The bottom plot of Fig. 7 illustrates the guarantee indicator, which is equal to 1 when the real position is included in the highest mass box and 0 otherwise. The figure shows that the method has erroneous measurements at 64 time steps, whereas it loses its guaranteed aspect at 14 of them. In fact, generally, when all observation disks have a nonempty intersection, even with the erroneous ones, the method loses its guaranteed aspect. For instance, the left plot of Fig. 8 shows the anchors disks obtained at $t=13 \mathrm{~s}$. The disks have a nonempty intersection, which will have the highest observation mass. The plot shows the focal elements of the mobility mass function as well as the estimated box. In contrast, the right plot of Fig. 8 shows the anchors disks given at $t=87 \mathrm{~s}$. It is obvious that the erroneous disk has an empty intersection with the correct observation disks and the mobility boxes. Then, when erroneous measurements have high reliability values but less than those of correct measurements, the method might lose its guaranteed aspect at time steps where the erroneous observation disks have nonempty intersections with the correct observation disks. In this case, the estimation boxes will not cover the real position of the node. However, other boxes, having smaller masses, are kept in the memory. Generally, these boxes cover the real position. Using them at the following time step reduces the propagation of error in future estimation steps.

Another configuration is then considered, in which correct and erroneous measurements have random reliability values chosen within $[0,1]$. In such a case, an erroneous measurement might have a high reliability exceeding reliability values of correct measurements. In this situation, the guaranteed aspect of the method is generally lost unless the erroneous measurement disk has an empty intersection with the mobility boxes. Then, the effectiveness of the method depends on the way the reliability values are assigned to measurements. In cases where good measurements have reliability values higher than 0.5 and erroneous ones have reliability values less than 0.5 , the localization result is always guaranteed. In other situations, the guaranteed aspect depends on the positioning of anchors, the previous estimated boxes, and the reliability values of measurements. However, since the method computes several focal elements at every time step, a wrong estimation at a specific instant is generally not efficient at following steps, which confirms the importance of the saving of several focal elements in the memory.

\section{Comparison With a Monte Carlo-Based Method}

In this paragraph, the robust localization (RL) method is compared with the Monte Carlo boxed localization (MCB) proposed in [13]. MCB is an anchor-based method proposed for environments where all measurements are supposed to be valid. It employs the Monte Carlo sequential approach given in [15]. Involving connectivity measurements, the MCB method generates a fixed number of positions, called particles, to cover the solution area. All particles are then kept in the memory to be used in the following time step. In case of empty intersection of observation constraints, the MCB method satisfies the mobility model only. To compare the RL method to the MCB method, the same simulated data as in Section V-A are used first. In particular, ten anchors are randomly deployed, four of them being within the vicinity of the node. The others are chosen within the ring centered on the node and having $r$ and $2 r$ as radii. The communication range $r$ is set to $10 \mathrm{~m}$ as well. All sensors are then moving using the group mobility paths, as shown previously. To generate erroneous measurements, a Gaussian noise with a zero mean and a variance equal to 

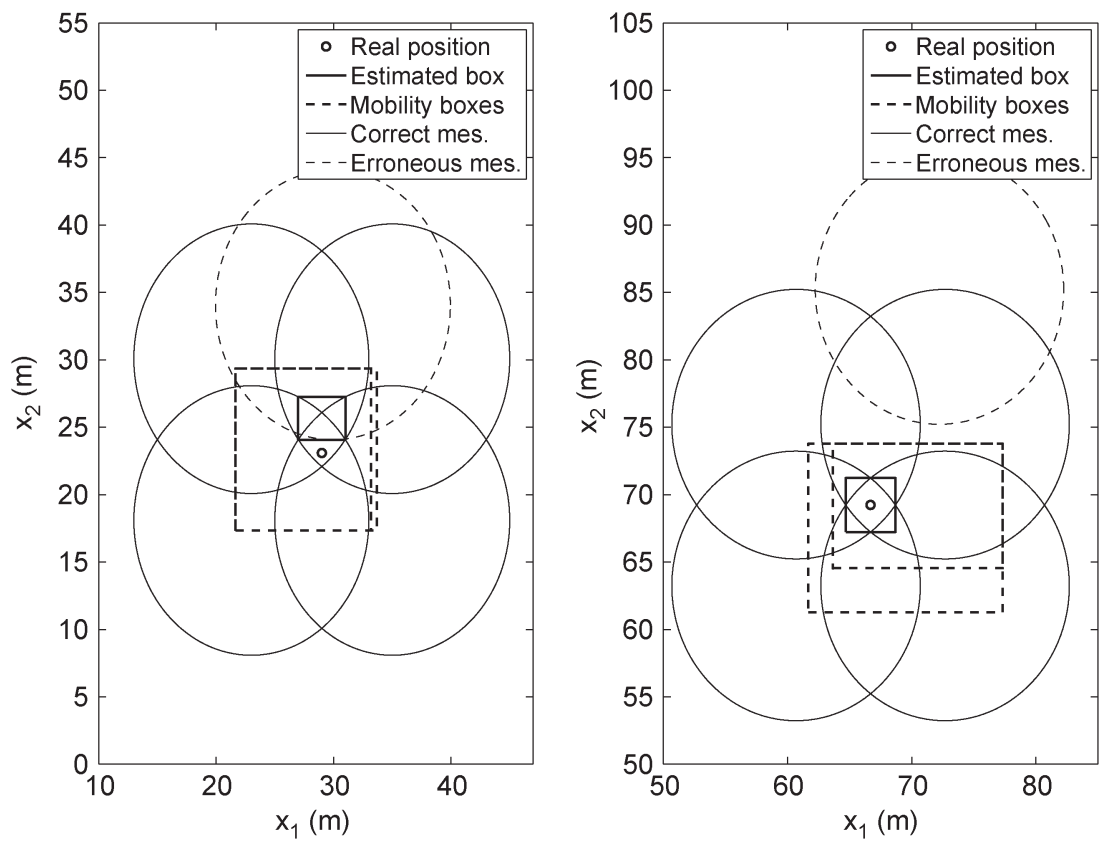

Fig. 8. Observation disks at time steps $t=13 \mathrm{~s}$ on the left plot and $t=87 \mathrm{~s}$ on the right plot.

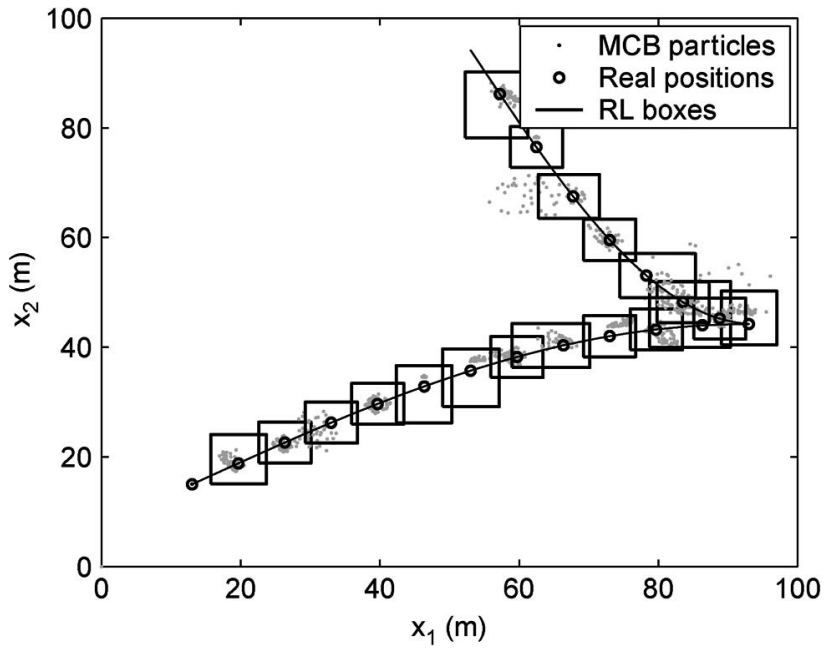

Fig. 9. Estimated boxes obtained with the RL method and the particles obtained with the MCB method with ten anchors in the network.

$4 \mathrm{dBm}^{2}$ is added to the correct RSSIs values. Then, regarding the RL method, measurements are assigned high reliability values if they are correct and low values otherwise. For the MCB method, 50 particles are generated at each time step. The maximal number of the iterations allowed to have these particles is set to 100. Fig. 9 shows the particles and the estimated boxes obtained using MCB and RL methods, respectively.The average computation times of both methods are equal to 0.02433 and $0.12714 \mathrm{~s}$ per time step, respectively. Therefore, the RL method is less consuming in terms of computation time compared to the MCB method. Let the estimation error for MCB be the distance between the barycenter of all particles and the real position. Then, the average estimation errors are equal to $0.4973 \mathrm{~m}$ for RL and $1.7646 \mathrm{~m}$ for MCB. Consequently, our method ensures a substantial gain in both estimation accuracy and computation time compared to the MCB method, particularly in imperfect environments. In fact, the MCB method

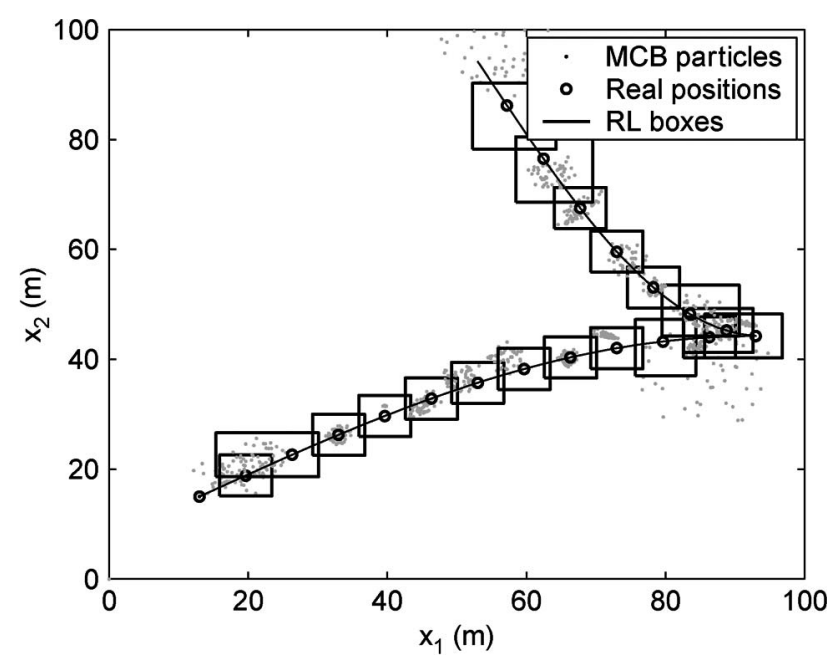

Fig. 10. Estimated boxes obtained with the RL method and the particles obtained with the MCB method with 20 anchors in the network.

overlaps all measurements, regardless of their reliability values, to define the estimated particles. If the resulting overlap region is empty, it considers the mobility constraint only. The presence of erroneous measurements leads to inaccurate overlap regions, yielding inaccurate estimates, which increases the estimation error. On the other hand, the generation process of 50 particles, needing several iterations, at each time step is high consuming in terms of computation time, compared with the interval-based estimation used in the RL method.

A comparison of both methods is then performed using 20 anchors, spread in the whole deployment area. The simulation is performed in the same conditions of error generation and reliability assignment as previously. Fig. 10 shows the particles and the boxes obtained with both methods. In this case, the average computation times per time step are equal to 0.02823 and $0.15834 \mathrm{~s}$, respectively, for RL and MCB methods. The average estimation errors are given by 0.4356 


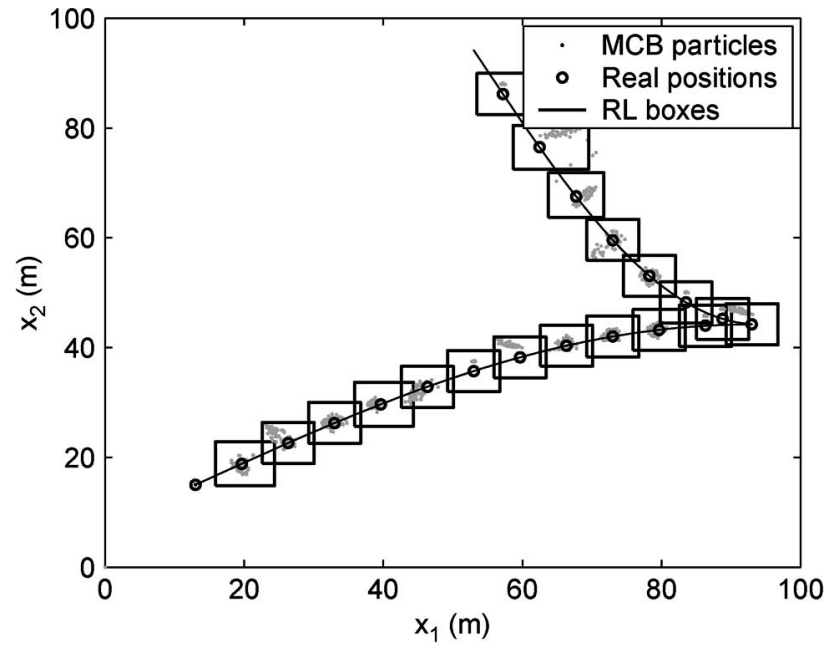

Fig. 11. Estimated boxes obtained with the RL method and the particles obtained with the MCB method with 30 anchors in the network.

TABLE IV

Comparison OF the RL Method to THE MCB Method USING DIFFERENT NUMBERS OF ANCHORS

\begin{tabular}{|l|c|c|c|c|}
\hline Total number of anchors & 10 anchors & 20 anchors & 30 anchors \\
\hline Computation times & RL & $0.02433 \mathrm{~s}$ & $0.02823 \mathrm{~s}$ & $0.03026 \mathrm{~s}$ \\
\cline { 2 - 5 } & MCB & $0.12714 \mathrm{~s}$ & $0.15834 \mathrm{~s}$ & $0.21637 \mathrm{~s}$ \\
\hline \multirow{2}{*}{ Estimation errors } & RL & $0.4973 \mathrm{~m}$ & $0.4356 \mathrm{~m}$ & $0.3905 \mathrm{~m}$ \\
\cline { 2 - 5 } & MCB & $1.7646 \mathrm{~m}$ & $1.8304 \mathrm{~m}$ & $2.1761 \mathrm{~m}$ \\
\hline Detected anchors & 3.05 & 3.15 & 3.31 \\
\hline Correct measurements & 2.74 & 2.75 & 2.8 \\
\hline Erroneous measurements & 0.31 & 0.4 & 0.51 \\
\hline
\end{tabular}

and $1.8304 \mathrm{~m}$, respectively. Now, if we consider 30 anchors spread in the whole deployment area, the average computation times per time step obtained with RL and MCB are equal to 0.03026 and $0.21637 \mathrm{~s}$, respectively, whereas the respective average estimation errors are given by 0.3905 and $2.1761 \mathrm{~m}$. Fig. 11 shows the particles and the boxes obtained with both methods with 30 anchors in the network. Table IV shows the total numbers of anchors, the average computation times and the average estimation errors obtained with both methods. The table shows the average numbers of detected anchors per time step as well as the average numbers of correct and erroneous measurements for each considered number of anchors. The results show that the computation times increase with the increase of the total number of anchors in the network in both RL and MCB methods. In fact, since both methods use all constraints at each time step, the increase of the number of detected anchors leads to the increase of the computation times. On the other hand, the estimation error decreases with the RL method with the increase of the total number of anchors, whereas it increases with the MCB method. In fact, MCB overlaps all constraints, including the erroneous ones, at each time step to define the estimated particles. Then, the increase of the number of erroneous measurements leads to the increase of the estimation error with the MCB method. Moreover, the RL method considers the reliability of each measurement. Since the correct measurements have high reliability and erroneous ones have low reliability, then the estimated boxes having the highest masses result generally from the overlap of the correct constraints. Thus, the increase of the number of correct

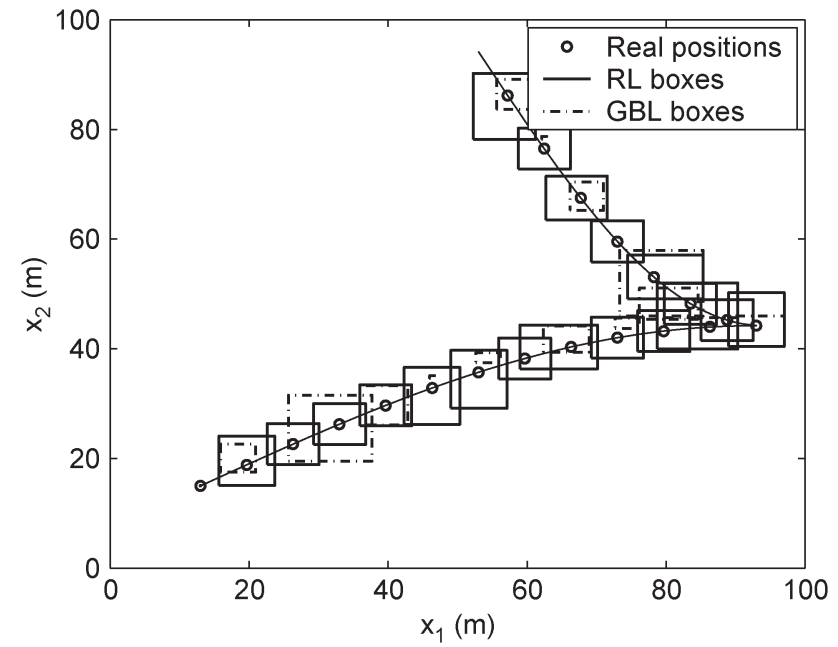

Fig. 12. Estimated boxes obtained with the RL method and the GBL method using ten anchors.

TABLE V

COMPARISON OF THE RL METHOD TO THE GBL METHOD USING DIFFERENT NUMBERS OF ANCHORS

\begin{tabular}{|l|c|c|c|c|}
\hline \multicolumn{2}{|c|}{ Total number of anchors } & 10 anchors & 20 anchors & 30 anchors \\
\hline \multirow{2}{*}{ Computation times } & RL & $0.02527 \mathrm{~s}$ & $0.02823 \mathrm{~s}$ & $0.03026 \mathrm{~s}$ \\
\cline { 2 - 5 } & GBL & $0.00577 \mathrm{~s}$ & $0.0064 \mathrm{~s}$ & $0.0071 \mathrm{~s}$ \\
\hline \multirow{2}{*}{ Estimation errors } & RL & $0.4973 \mathrm{~m}$ & $0.4356 \mathrm{~m}$ & $0.3905 \mathrm{~m}$ \\
\cline { 2 - 5 } & GBL & $1.4819 \mathrm{~m}$ & $1.6261 \mathrm{~m}$ & $1.7675 \mathrm{~m}$ \\
\hline \multirow{2}{*}{ Lost of guarantee } & RL & 0 & 0 & 0 \\
\cline { 2 - 5 } & GBL & 27 & 29 & 33 \\
\hline
\end{tabular}

measurements leads to the increase of the accuracy of the RL method, leading to the decrease of the estimation error, which agrees with the simulated results.

\section{Comparison With an Interval-Based Method}

In this section, the RL method is compared to the guaranteed boxed localization (GBL) proposed in [17]. Handling interval data, the GBL method is developed for perfect circumstances where measurements are supposed to be true. At each time step, it iterates both the mobility and connectivity equations in the Waltz algorithm to compute the solution box. It is worth noting that the GBL method uses only the mobility model at time steps where the observation disks have an empty intersection. Similarly to the simulations illustrated in Section V-C, the GBL and the RL methods are first compared using ten anchors. Their performances are then illustrated using 20 and 30 anchors. In all cases, erroneous measurements are generated by adding a zero-mean Gaussian noise with a variance of $4 \mathrm{dBm}^{2}$ to the RSSIs. Fig. 12 illustrates the estimated boxes obtained with both RL and GBL in the case of ten anchors. The plot shows that the GBL boxes are either too small but not including the real positions or too large using only the mobility equation. The guaranteed aspect is lost at 27 time steps with GBL, whereas it is maintained at all time steps with RL. The average computation time, however, increases from $0.00577 \mathrm{~s}$ with GBL to $0.02527 \mathrm{~s}$ with RL. In contrast, the average estimation error decreases from $1.4819 \mathrm{~m}$ with GBL to $0.4973 \mathrm{~m}$ with RL. Table V shows the average computation times, the average estimation errors, and the number of time 
steps where the guaranteed aspect is lost in both methods using 10,20 , and 30 anchors. It is obvious that the increase of the estimation error is related to the lost of guarantee. The results show that the proposed method is more efficient than the GBL method in terms of accuracy at the cost of the computation time. In fact, similarly to the MCB method, the GBL method uses all constraints, regardless of their reliability values. Since erroneous measurements create generally regions not including the exact position, using all measurements in GBL might lead to estimated boxes not covering the correct positions. For this reason, the estimation errors of the GBL method is higher than those of the RL method. It is worth noting that the RL method achieves several combinations at each time step leading to multiple focal elements. This is why it is more consuming than the GBL method in terms of computational time.

\section{CONCLUSiON}

This paper has proposed an original method for sensor localization in imperfect circumstances. The method is developed for environments where erroneous observations may occur. The robustness of the method remains in the way that it makes use of the reliability of the measured data to define the solution. Using both belief and interval theories, the method proposes more than one possible position at every time step, each of which having a specific weight. Computed positions are 2-D intervals, whose centers define the punctual estimated positions of the nodes. The effectiveness of the method is evaluated using simulations on mobile sensors that have group trajectories. Experimental results show that our method outperforms the existing methods for perfect environments when erroneous data are collected. Future works will handle the estimation of the observations reliability. Moreover, one can define the localization problem in different imperfect scenarios where, for instance, only the maximal number of erroneous measures is supposed to be known.

\section{REFERENCES}

[1] I. F. Akyildiz, W. Su, Y. Sankarasubramaniam, and E. Cayirci, "Wireless sensor networks: A survey," Comput. Netw., vol. 38, no. 4, pp. 393-422, Mar. 2002.

[2] H. Karl and A. Willig, "A short survey of wireless sensor networks," Tech. Univ. Berlin, Telecommun. Netw. Group, Berlin, Germany, Tech. Rep. TKN-03-018, 2003.

[3] M. Moges and T. Robertazzi, "Wireless sensor networks: Scheduling for measurement and data reporting," IEEE Trans. Aerosp. Electron. Syst., vol. 42, no. 1, pp. 327-340, Jan. 2006.

[4] A. Czubak and J. Wojtanowski, "On applications of wireless sensor networks," in Internet-Technical Development and Applications. Heidelberg, Germany: Springer Berlin, 2009, pp. 91-99.

[5] W. S. Conner, J. Heidemann, L. Krishnamurthy, X. Wang, and M. Yarvis, "Workplace applications of sensor networks," in Wireless Sensor Networks: A Systems Perspective, N. Bulusu and S. Jha, Eds. Norwood, MA: Artech House, 2004.

[6] N. Xu, A Survey of Sensor Network Applications. [Online]. Available: http://citeseerx.ist.psu.edu/viewdoc/summary?doi=10.1.1.131.9647

[7] S. Kumar, F Zhao, and D. Shepherd, "Special issue on collaborative information processing," IEEE Signal Process. Mag., vol. 19, no. 2, pp. 13-14, Mar. 2002.

[8] "Special issue on self-organizing distributed collaborative sensor networks," IEEE J. Sel. Areas Commun., vol. 23, no. 4, pp. 689-872, Apr. 2005.

[9] B. Chen, L. Tong, and P. Varshney, "Special issue on distributed signal processing in sensor networks," IEEE Signal Process. Mag., vol. 23, no. 4, pp. 16-26, Jul. 2006.
[10] B. Hofmann-Wellenhof, H. Lichtenegger, and J. Collins, Global Positioning System: Theory and Practice. New York: Springer-Verlag, 1994.

[11] A. El-Rabbany, Introduction to GPS: The Global Positioning System. Norwood, MA: Artech House, 2002.

[12] L. Hu and D. Evans, "Localization for mobile sensor networks," in Proc. 10th Annu. Int. Conf. MobiCom, 2004, pp. 45-57.

[13] A. Baggio and K. Langendoen, "Monte-Carlo localization for mobile wireless sensor networks," in Proc. 2nd Int. Conf. MSN, 2006, pp. 317-328.

[14] Y. Jiyoung, Y. Sungwon, and C. Hojung, "Multi-hop-based Monte Carlo localization for mobile sensor networks," in Proc. 4th Аnnu. IEEE Commun. Soc. Conf. SECON, 2007, pp. 162-171.

[15] A. Doucet, S. Godsill, and C. Andrieu, "On sequential Monte-Carlo sampling methods for Bayesian filtering," Statist. Comput., vol. 10, no. 3, pp. 197-208, Jul. 2000

[16] F. Mourad, H. Snoussi, F. Abdallah, and C. Richard, "Guaranteed boxed localization in manets by interval analysis and constraints propagation techniques," in Proc. IEEE Globecom, 2008, pp. 1-5.

[17] F. Mourad, H. Snoussi, F. Abdallah, and C. Richard, "Anchor-based localization via interval analysis for mobile ad-hoc sensor networks," IEEE Trans. Signal Process., vol. 57, no. 8, pp. 3226-3239, Aug. 2009.

[18] F. Mourad, H. Snoussi, F. Abdallah, and C. Richard, Localisation par intervalles basée sur les ancres et les non-ancres dans les réseaux de capteurs mobiles. Toulouse, France: GRETSI, 2009.

[19] R. E. Moore, Methods and Applications of Interval Analysis. Philadelphia, PA: SIAM, 1979.

[20] R. B. Kearfott and V. Kreinovich, Applications of Interval Computations. Norwell, MA: Kluwer, 1996.

[21] E. Hansen and G. W. Walster, Global Optimization Using Interval Analysis, 2nd ed. New York: Marcel Dekker, 2004

[22] L. Jaulin, M. Kieffer, O. Didrit, and E. Walter, Applied Interval Analysis. New York: Springer-Verlag, 2001.

[23] F. Caron, M. Davy, E. Duflos, and P. Vanheeghe, Fusion de capteurs potentiellement défaillants par filtrage particulaire. Toulouse, France: GRETSI, 2005.

[24] A. Dempster, "Upper and lower probabilities induced by a multivalued mapping," Ann. Math. Statist., vol. 38, no. 2, pp. 325-339, 1967.

[25] G. Shafer, A Mathematical Theory of Evidence. Princeton, NJ: Princeton Univ. Press, 1976.

[26] G. Shafer and J. Pearl, Readings in Uncertain Reasoning. San Mateo, CA: Morgan Kaufman, 1990.

[27] P. Smets and R. Kennes, "The transferable belief model," Artif. Intell., vol. 66, no. 2, pp. 191-234, Apr. 1994.

[28] R. R. Yager and L. Liu, Eds., Classic Works of the Dempster-Shafer Theory of Belief Functions. Berlin, Germany: Springer-Verlag, 2008, ser. Studies in Fuzziness and Soft Computing.

[29] X. Hong, M. Gerla, G. Pei, and C.-C. Chiang, "A group mobility model for ad hoc wireless networks," in Proc. 2nd ACM Int. Workshop MSWiM, 1999, pp. 53-60.

[30] T. Camp, J. Boleng, and V. Davies, "A survey of mobility models for ad hoc network research," Wirel. Commun. Mobile Comput.-Special Issue on Mobile Ad Hoc Networking: Research, Trends and Applications, vol. 2, no. 5 , pp. 483-502, 2002

[31] A. Medeisis and A. Kajackas, "On the use of the universal Okumura-Hata propagation prediction model in rural areas," in Proc. IEEE Veh. Technol. Conf., 2000, vol. 3, pp. 1815-1818.

[32] Z. Nadir, N. Elfadhil, and F. Touati, "Pathloss determination using Okumura-Hata model and spline interpolation for missing data for Oman," in Proc. World Congr. Eng., London, U.K., Jul. 2008, vol. 1.

[33] T. Denux, "Analysis of evidence-theoretic decision rules for pattern classification," Pattern Recognit., vol. 30, no. 7, pp. 1095-1107, Jul. 1997.

[34] B. Ristic and P. Smets, "Target identification using belief functions and implication rules," IEEE Trans. Aerosp. Electron. Syst., vol. 41, no. 3, pp. 1097-1103, Jul. 2005.

[35] S. Petit-Renaud and T. Denux, "Nonparametric regression analysis of uncertain and imprecise data using belief functions," Int. J. Approx. Reason., vol. 35, no. 1, pp. 1-28, Jan. 2004.

[36] R. Yager, "Cumulative distribution functions from Dempster-Shafer belief structures," IEEE Trans. Syst., Man, Cybern. B, Cybern., vol. 34, no. 5, pp. 2080-2087, Oct. 2004.

[37] H. Xu, Y.-T. Hsia, and P. Smets, "Transferable belief model for decision making in valuation-based systems," IEEE Trans. Syst., Man, Cybern. A, Syst., Humans, vol. 26, no. 6, pp. 698-707, Nov. 1996.

[38] P. Vannoorenberghe, "Un état de l'art sur les fonctions de croyance appliquées au traitement de l'information," Inf. Interaction Intell., vol. 3, no. 2, pp. 9-45, 2003. 


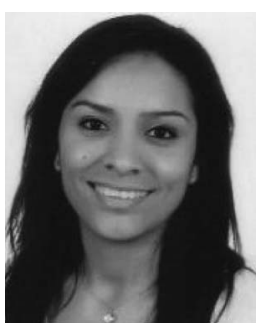

Farah Mourad was born in Tripoli, Lebanon, in 1984. She received the Diploma in electrical engineering from the Lebanese University, Tripoli, in 2006 and the Master de recherche and Ph.D. degrees sité de Technologie de Troyes, Troyes, France, in 2007 and 2010, respectively. Delaunay-LM2S (UMR STMR CNRS 6279), Université de Technologie de Troyes. Her research interests include the localization of mobile sensors and the tracking of targets in mobile sensor networks. in systems optimization and safety from the Univer-

She is currently with the Institut Charles

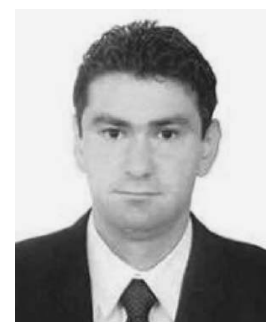

Fahed Abdallah was born in Lebanon on August 18, 1976. He received the Dipl.Ing. and M.S. degrees in electrical and computer engineering from the Lebanese University, Beirut, Lebanon, in 1999 and 2000 , respectively, the Ph.D. degree in electrical and computer engineering from Troyes University of Technology, Troyes, France, in 2004, and the HdR degree from the University of Technology of Compiègne, Compiègne, France, in 2010.

Since 2005, he has been an Associate Professor with the Laboratoire HEUDIASYC (UMR CNRS 6599), Université de Technologie de Compiègne. His current research interests include state estimation for dynamic models based on multisensor fusion, statistical estimation and decision theories, pattern recognition, and belief function theory.

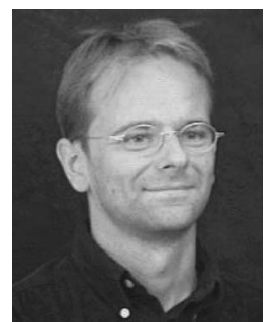

Cédric Richard (S'98-M'01-SM'07) was born in Sarrebourg, France, on January 24, 1970. He received the Dipl.-Ing. and M.S. degrees in electrical and computer engineering and the Ph.D. degree in electrical and computer engineering from the University of Technology of Compiègne, Compiègne, France, in 1994 and 1998, respectively.

From 1999 to 2003, he was an Associate Professor with the University of Technology of Troyes (UTT), Troyes, France. From 2003 to 2009, he was a Full Professor with the Institut Charles Delaunay (CNRS 1976. He received the Diploma in electrical engineering from the Ecole Supérieure d'Electricité (Supelec), Gif-sur-Yvette, France, in 2000, the DEA and $\mathrm{Ph} . \mathrm{D}$. degrees in signal processing from the University of Paris-Sud, Orsay, France, in 2000 and 2003, respectively, and the HdR degree from the University of Technology of Compiègne, Compiègne, France, in 2009.

Between 2003 and 2004, he was a Postdoctoral Researcher with the Institut de Recherches en Communications et Cybernétiques de Nantes, Nantes, France. He has spent short periods as a Visiting Scientist at the Brain Science Institute, RIKEN, Tokyo, Japan, and Olin Neuropsychiatry Research Center at the Institute of Living, Hartford, CT. Between 2005 and 2010, he was an Associate Professor with the University of Technology of Troyes, Troyes, France, where he has been appointed as a Full Professor within the Institut Charles Delaunay-LM2S (UMR STMR CNRS 6279) since September 2010. He is in charge of the regional research program System Security and Safety (S3) of the CPER 2007-2013 and the CapSec platform (wireless embedded sensors for security). He is the Principal Investigator of an ANR-Blanc project (mv-EMD), which is a CRCA project (new partnership and new technologies), and a GDRISIS young researcher project. He is a partner of many ANR projects, GIS, strategic UTT programs.

Dr. Snoussi received the Prime d'Encadrement Doctoral et de Recherche 2008-2012 national doctoral and research supervising award.
FRE 2848) at the UTT, and the supervisor of a group consisting of 60 researchers and Ph.D.s. In winter 2009 and autumn 2010, he was a Visiting Researcher with the Department of Electrical Engineering, Federal University of Santa Catarina, Florianòpolis, Brazil. Since September 2009, he has been a Full Professor at Laboratoire FIZEAU (UMR CNRS 6525, Observatoire de la Côte d'Azur), Université de Nice Sophia-Antipolis, Nice, France. He has served as an Associate Editor for the EURASIP Signal Processing Magazine since 2009. He is the author of over 100 papers. His current research interests include statistical signal processing and machine learning.

Dr. Richard has been a Junior Member of the Institut Universitaire de France since October 2010. He is a member of GRETSI Association Board and of the EURASIP Society. Since 2005, he has been a member of the Board of the federative CNRS research group ISIS on Information, Signal, Images, and Vision. He has served as an Associate Editor for the IEEE TRANSACTIONS on Signal Processing since 2006. He was the General Chair of the 21st francophone conference GRETSI on Signal and Image Processing that was held in Troyes, France, in 2007 and of the IEEE International Workshop on Statistical Signal Processing that will be held in Nice in 2011. In 2009, he was nominated as Liaison Local Officer for EURASIP and as member of the Signal Processing Theory and Methods Technical Committee of the IEEE Signal Processing Society. He received Best Paper Award for "Solving the pre-image problem in kernel machines: A direct method" at the 2009 IEEE Workshop on Machine Learning for Signal Processing, along with P. Honeine. 